\title{
Knowledge of oral health of nursing staff caring for disadvantaged older people
}

\author{
Jori A. Reigle ${ }^{* 1}$, Karyn Holm² \\ ${ }^{1}$ University of Michigan-Flint, Flint, Michigan, United States \\ ${ }^{2}$ DePaul University, Chicago, Illinois, United States
}

Received: August 4, 2015

DOI: $10.5430 /$ jnep.v6n1p31

\author{
Accepted: September 8, $2015 \quad$ Online Published: October 12, 2015
}

URL: http://dx.doi.org/10.5430/jnep.v6n1p31

\begin{abstract}
Poor oral health plagues those who are economically disadvantaged and it is older people who are particularly vulnerable. Healthcare professionals, notably nursing staff, can contribute to the reduction of oral health disparities in older men and women. The purpose of this study was to describe an oral health education program and evaluate its effect on oral health knowledge of nursing staff serving economically disadvantaged older people in a religiously affiliated nursing home. The study was structured by a one-group pretest-posttest design to determine whether a change occurred in the oral health knowledge following participation by nursing staff in an oral health education program. Knowledge of Oral Health $(\mathrm{KOH})$ questionnaire, used as both pretest and posttest, was developed to achieve the purpose of the study and knowledge improved significantly. The KOH posttest displayed overall higher scores $(M=7.50, \mathrm{SD}=1.701)$ than the pretest scores $(\mathrm{M}=5.40, \mathrm{SD}=1.569)$. The $\mathrm{KOH}$ score increased by an average of 2.10 points with $\mathrm{SD}=1.619$ and $95 \%$ confidence interval, 1.342, 2.858. The intervention effect was large and statistically significant, $t(19)=5.801, p<.001$. Future research should be directed toward refining the KOH questionnaire, determining its application internationally, and directing the oral health education program to include not only nursing staff but older people as well.
\end{abstract}

Key Words: Oral health, Knowledge, Nursing, Education, Older

\section{INTRODUCTION}

Oral health is a major problem for people of disparate populations of all ages living in developed or developing countries. ${ }^{[1]}$ In The World Oral Health Report, Petersen ${ }^{[1]}$ reports the "greatest burden of oral diseases is on disadvantaged and socially marginalized populations." The Institute of Medicine and National Research Council Committee on Oral Health Access to Services calls for exploring role expansion of non-dental healthcare professionals, notably nursing staff, because they are in optimal situations to contribute to the reduction of lack of access to care which increases oral health disparities in the United States. ${ }^{[2]}$ "Adequate oral health manpower and primary health personnel trained in oral health care for older people will ensure appropriate oral health care as an integral part of primary health services."[3] In nursing homes in the United States, appropriate oral care for some patients is completely dependent on the nursing staff, which is dependent on their oral health knowledge. For independent patients, oral health promotion and teaching to improve the knowledge of older people will improve their attitudes, oral self-care, and health as found in an international study of a community oral health promotion program. ${ }^{[4]}$ The purpose of this quantitative quasi-experimental study is to describe an oral health education program for nursing staff and evaluate its effect on oral health knowledge of nursing staff serving economically disadvantaged older people in a

*Correspondence: Jori A. Reigle; Email: jorireigle @gmail.com; Address: University of Michigan-Flint, Flint, Michigan, United States. 
religiously affiliated nursing home.

\subsection{Background and literature}

The literature review examines the relationships between oral health and overall health, oral health and its social impact, and oral health and disadvantaged older people. Additionally, the concept of oral health knowledge and oral health related to nursing home policies and nursing staff were explored. An electronic literature search was performed using the following databases: CINAHL, PubMed, Google Scholar and ProQuest Nursing \& Allied Health Source. Combinations of the terms "oral health", "dental", "oral hygiene", "nurs*," "knowledge", "elderly" and "economically disadvantaged" were used. Additional articles were found in reference sections of articles previously searched.

\subsubsection{Oral health and overall health}

Poor oral health is a major public health concern. The World Health Organization (WHO) recognizes older people as a target population for oral health and non-communicable disease interventions. $^{[3,5]}$ Prominent non-communicable diseases with modifiable risk factors identified by the WHO include cardiovascular disease, diabetes, cancer, and chronic obstructive pulmonary disease and are commonly affecting the older population with association to poor oral health. ${ }^{[3,5]}$ Modifiable risk factors of these non-communicable diseases are similar to modifiable risk factors of many oral diseases. ${ }^{[1,6]}$ The process of aging also presents specific concerns related to the oral health of older people including caries, tooth loss (edentulism), difficulty chewing, reduced salivation, oral cancer, xerostomia (dry mouth), craniofacial pain and discomfort, gingival overgrowth, and oro-facial bone resorption. ${ }^{[1]}$ Although the rate of edentulism in this target population is decreasing as more are retaining more teeth, ${ }^{[7]}$ there is still concern that older people are at risk for oral health problems. ${ }^{[8]}$

\subsubsection{Social impact of oral health}

To support the common assumption that there are negative social effects for edentulous individuals, Willis, Esqueda, and Schacht ${ }^{[9]}$ studied the perception of participants viewing black and white photos of individuals with obvious missing front teeth. The study found that edentulous individuals are ranked lower on many social characteristics in comparison with non-edentulous individuals. ${ }^{[9]}$ Photographs of mouths with missing teeth are ranked lower on scales of attractiveness, health, and intelligence. ${ }^{[9]}$ Individuals with poor oral health report negative effects on their ability to socially interact and engage in intimacy. ${ }^{[6,10]}$ Also regarding oral malodor, which is often present in poor oral health, researchers found negative psychosocial problems such as embarrassment. ${ }^{[9]}$

\subsubsection{Oral health and the disadvantaged older people}

Older people in nursing homes are vulnerable to poor oral and overall health. ${ }^{[11]}$ They are likely prescribed multiple medications, which increase the risk of poor oral health, ${ }^{[1]}$ but also experience declining independence that negatively impacts quality of life. Side effects of these medications may include reduced salivary flow, xerostomia, difficulty tasting, chewing, and smelling; some of which increase the risk of dental caries and periodontal disease. ${ }^{[5]}$ The U.S. Surgeon General report states that at least one of the medications likely prescribed to older people will have side effects contributing to poor oral health with the most common being dry mouth from reduced salivary flow. ${ }^{[10]}$ Locker, Matear, Stephens, and Jokovic ${ }^{[12]}$ surveyed older participants and found that one third of the participants rated their personal oral health as "fair" or "poor" and these participants had lower morale, higher life stress, and lower levels of overall life satisfaction.

Disparities exist amongst older people of higher-income compared to those who are economically disadvantaged. Griffin et al. ${ }^{[6]}$ reviewed data collected between 2005-2008 from the National Health and Nutrition Examination Survey (NHANES) and found four major themes related to oral health status and disease of the groups of older populations examined (50-64, 65-74, and more than 75 years old). The review supported previous studies that poor overall and oral health are linked but also economically disadvantaged older people are more likely to have untreated oral health problems. ${ }^{[6]}$ Nursing staff in the United States today are more commonly caring for an older adult and serve in a helping role to lower the negative impact of poor oral health and reduce disparities in populations such as the disadvantaged older people.

\subsubsection{Oral health knowledge}

Evaluation of the oral health knowledge of low-income Baltimore adults was accomplished using the Baltimore Health Literacy and Oral Health Knowledge Project questionnaire. ${ }^{[13]}$ The questionnaire was designed to assess knowledge of basic oral health and the prevention and management of dental caries, periodontal disease, and oral cancer. ${ }^{[13]}$ The authors define oral health knowledge as knowledge of these four broad topics in association with health literacy. ${ }^{[13]}$ Participants were primarily African-American women, ages 45-64 years old, receiving less than or equal to an income of $\$ 25,000$ a year with 12 years of education. ${ }^{[13]}$ Results of the cross-sectional population-based study indicated that most understood basic techniques to prevent tooth decay; however, results were mixed in their knowledge of prevention and management of gingivitis and periodontitis. ${ }^{[13]}$ Nearly $75 \%$ of participants incorrectly believed that oral hygiene practice 
includes the use of a "medium" or "hard" bristle toothbrush and less than $50 \%$ correctly answered that smoking cigarettes and diabetes are risk factors for poor oral health and periodontal disease. ${ }^{[13]}$ An alarming majority of participants believed that plaque was made up of food and not composed of germs that can cause gingivitis and periodontitis. ${ }^{[13]}$ Oral health knowledge in this study is basic knowledge of the relationship between oral health and overall health, maintenance of oral health, and prevention and management of oral disease.

\subsubsection{Oral health and nursing home policies}

It is the responsibility of nursing staff to ensure that adequate oral care is provided in inpatient settings where dental professionals are lacking or only present for short durations. ${ }^{[14]}$ For some patients, oral care is completely dependent on nursing staff $^{[14]}$ and for other patients, oral health promotion and teaching is necessary to encourage good oral health behaviors.

The United States Government Code of Federal Regulations 483.25(a)(3) for long-term care facilities requires that "a resident who is unable to carry out activities of daily living receives the necessary services to maintain good nutrition, grooming, and personal and oral hygiene." ${ }^{[15]}$ However, nursing homes may have a limited capacity to deliver the level of oral health care needed to maintain oral health and prevent disease of its residents. ${ }^{[10]}$ "Oral hygiene" is not specifically outlined and facilities lack policies to guide nursing staff. ${ }^{[16]}$ This results in substandard oral health of older people as reported by Coleman and Watson ${ }^{[8]}$ in a study that found very little time was spent on oral health care including brushing teeth and tongue, flossing, evaluating the oral cavity as well as not wearing clean gloves while providing oral care.

A multidisciplinary approach is key to improving the oral health of older people and when the duties are to be fulfilled by the nursing staff then the policies and guidelines regarding care should be driven and received by nursing staff. ${ }^{[7]}$ Blinkhorn et al. ${ }^{[7]}$ used focus groups to identify three barriers to methods to improve oral health of older residents, some with mental health problems, in a nine ward hospital. Two of these barriers include lack of policies or guidelines and lack of knowledge by nursing staff. ${ }^{[7]}$ A solution included an oral hygiene protocol designed by nurses which included use of equipment easily available in an "oral hygiene trolley" utilized alongside medication administration and an oral health education program to introduce oral health knowledge evaluated through pretests and posttests. ${ }^{[7]}$ Evaluation of effects on oral health of residents was measured at the beginning of the study, three months, and 12 months later where findings show improvements in oral health and cleanliness. ${ }^{[7]}$

Published by Sciedu Press
Oral health knowledge of nursing staff improved between pretests and posttests taken 16 weeks after the education program. ${ }^{\text {[7] }}$ In addition to being supported by nurses and ward residents, the interventions coincided with a decrease in antibiotic prescriptions, a factor not initially intended for study. ${ }^{[7]}$

Policy is needed to guide oral health care by nursing staff as well as education to improve their knowledge and process of providing oral health care to patients. Too little time is devoted to oral health and disease topics in the education of non-dental health professionals as reported by the USDHHS. ${ }^{[10]}$ Mynors-Wallis and David ${ }^{[14]}$ conducted a study assessing the oral health knowledge of nurses working with older people before a dental talk intervention and one month later. They conclude that more questions on the modernized version of Rak and Warren's ${ }^{[17]}$ assessment of dental and mouth care knowledge involving the day-to-day care of patients were answered correctly and theoretical questions were answered incorrectly less often. ${ }^{[14]}$ There was a statistically significant $(p<.0001)$ increase of $25 \%$, from $53 \%$ to $78 \%$, in the mean of number of correct answers on the questionnaire given to nurses before a dental talk and administered again one month later. ${ }^{[14]}$ Mynors-Wallis and Davis $^{[14]}$ also reported that nurses find oral health care as disagreeable because of patients' unwillingness to participate. Therefore, oral health education is necessary to change perceptions about oral health care.

\subsubsection{Oral health and nursing staff}

In Stockholm, a study reported the experiences of nursing home staff taking part in a three-step dental hygiene education program. ${ }^{[18]}$ The steps include hands-on training on electric toothbrush technique, small discussion groups and a theoretical lecture. Their results included the improvement in the belief by nursing staff that they have adequate knowledge to carry out daily oral care and a reduction in their perception of oral hygiene as unpleasant. ${ }^{[18]}$ They also noted that the experiences of the described dental hygiene education program for staff serves as a starting point for the development of an education model and both quantitative and qualitative studies to support its effectiveness. ${ }^{[18]}$ They later repeated the education program for nursing home staff noting improvement of oral hygiene of older people; however, perceptions and attitudes towards oral care continue to be a need to address. ${ }^{[19]}$

A randomized controlled trial conducted by Frenkel, Harvey, and Needs ${ }^{[20]}$ found that an oral health education program improved attitudes about oral health and knowledge of nursing home staff improved to $85.4 \%$ from a baseline score of $76.3 \%$ in a quasi-experimental design using a pretest and posttest 
following intervention. A follow-up self-reported survey conducted 7.5 months later also showed that staff continued to use the education resources and oral hygiene products provided by Parsons. ${ }^{[21]}$ However, not all oral health education programs are successful in changing nursing staff's perceptions, knowledge, and behaviors of oral health care provided to patients. Gammack and Pulisetty ${ }^{[16]}$ examined the effectiveness of an oral health education program but unfortunately the 30-minute multimedia education program found no statistical significance in the nurses' quality of oral health care before and after the intervention. More disappointingly, the average length of time spent providing oral health care by brushing teeth, gums, and dentures of the residents actually decreased from 58 to 52 seconds. ${ }^{[16]}$

\subsection{Summary}

The literature demonstrates a pattern of results and recommendations addressing the need to describe and evaluate an oral health education program for nursing staff. Findings of published studies show that more efficacious programs that are repeated throughout a longer specified time period are needed in order to help change oral health behaviors. Dental and non-dental health professionals should continue to examine research addressing the best method of improving the oral health knowledge of nursing staff with the purpose of improving their perceptions, attitudes, and behaviors regarding correct oral care for their older residents. Therefore, the present study was designed to contribute to the growing body of research.

\section{METHODS}

\subsection{Purpose and research design}

The purpose of this quantitative quasi-experimental study was to describe an oral health education program for nursing staff caring for disadvantaged older people and evaluate its effect on their oral health knowledge using the $\mathrm{KOH}$ questionnaire developed and tested by the authors. Figure 1 shows the research process using a one-group pretest-posttest design to determine whether a change occurred in the oral health knowledge following participation in the oral health education program intervention.

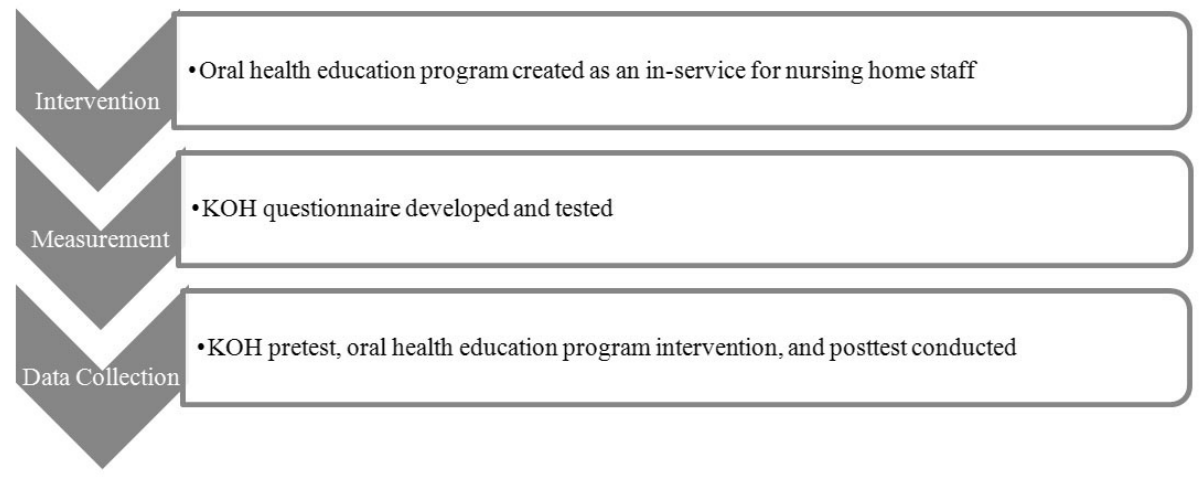

Figure 1. Sequence of study events

\subsection{Description of the oral health education program}

The intervention was an 18-minute oral health education program with a visual component of the Oral Health Knowledge for Nurses' Caring for the Disadvantaged Elderly PowerPoint presentation created by the researcher in the form of an in-service. The presentation topics included oral and systemic health, oral health problems common in older people, a review of common oral side effects of medications, and the introduction of the SMILE acronym. The SMILES acronym stands for Soft bristles, Morning and evening, Inner and outer, Lower and upper, Evaluate everyday. Some content was adapted from Smiles for Life: A National Oral Health Curriculum with permission from Melinda Clark, Smiles for Life editor, via e-mail after a request form was completed. The curriculum is an online resource that is available for dental and non-dental health professionals seeking to learn more information about oral health and has been supported in the literature because it "provides a knowledge framework for nurse faculty enrichment and competency development in oral health across the life cycle."[22] The eight courses can be completed for free continuing education contact hours through the New York University College of Nursing's Cen-

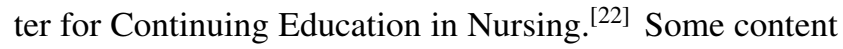
of the PowerPoint presentation comes from the researcher's knowledge gained as a Chicago area Albert Schweitzer fellow implementing an oral health promotion program with the underserved population utilizing the services of a local food pantry.

\subsection{Setting and sample}

The pretest, intervention, and posttest using the $\mathrm{KOH}$ questionnaire were offered to a convenience sample of consenting 
nursing staff providing direct care to disadvantaged older people. Data collection occurred in a small library environment during a regularly scheduled bimonthly one hour in-service. In addition to accessibility, the home welcomes education in-services as a means to maintain necessary clinical knowledge to provide optimum care for their patients. Twenty-two staff members were in attendance at the beginning of the in-service and two staff members arrived during the intervention but did not participate in the study portion for a total of twenty-four nursing staff in the study location. Twenty completed the study as one staff member withdrew because she was unable to see the slides without her glasses and a second participant did not identify the $\mathrm{KOH}$ questionnaire with the provided study code therefore data could not be linked appropriately. Participants included 15 nurse assistants, two restorative nurse aides, two registered nurses, and one licensed practical nurse.

\subsection{Knowledge of Oral Health $(\mathrm{KOH})$ measurement}

Knowledge of oral health of nursing staff was measured by administering the $\mathrm{KOH}$ questionnaire based upon the Smiles for Life: A National Oral Health Curriculum examination ${ }^{[23]}$ and the SMILE acronym tool constructed specifically to address the purpose of this study as a pretest and postest. Permission was given to use the Smiles for Life questions by the editor.

Table 1. Knowledge of Oral Health $(\mathrm{KOH})$ questionnaire

1) What type of toothbrush is best to use?

2) When is the best time to remind residents to perform oral self-care or perform oral care for dependent patients?

3) In a circular pattern, toothbrushing should include the following portions of the mouth:

4) What is a consequence of untreated dental cavities (tooth decay)?

5) Which of these classes of medications is NOT generally associated with decreased salivary flow?

6) What is the suggested common pathway linking chronic periodontitis and conditions such as diabetes, coronary artery disease and adverse pregnancy outcomes?

7) Which of the following statements is true regarding the oral health of elderly patients with dementia?

8) Risk factors for adult caries include all but which of the following?

9) Which of the following statements concerning xerostomia, or dry mouth, is not true?

10) How many teeth do adults normally have?

The $\mathrm{KOH}$ questionnaire contains ten questions with four multiple choice answer options, not shown in Table 1 for brevity, and only one correct answer. Questions 1-3 about the type of toothbrush, the times teeth should be brushed, and the portions of the mouth that should be brushed reflect the SMILE acronym taught during the intervention. The remaining questions were based on the Smiles for Life examination. A total score was calculated for both the pretest and posttest with a perfect score totaling 100 points. An unscored question at the end of the $\mathrm{KOH}$ questionnaire asked participants to "Please identify your nursing role below: A. nurse assistant, B. licensed practical nurse, C. registered nurse, D. advanced practice nurse and E. other, please specify."

\subsection{Validity and reliability}

The $\mathrm{KOH}$ questionnaire included questions created by the researcher and questions from the Smiles for Life: A National Oral Health Curriculum examination, which was designed to assess oral health knowledge of primary care physicians serving patients of all ages. ${ }^{[1]}$ The examination was developed by a Society of Teachers of Family Medicine Group (STFM) national steering committee of physicians and dentists, which

Published by Sciedu Press provides support to the content validity of the $\mathrm{KOH}$ questionnaire. The questions created by the researcher assess the understanding of the SMILE acronym tool. The $\mathrm{KOH}$ questionnaire was reviewed and supported by the project coordinator of the Chicago Community Oral Health Forum and co-author of the Chicago Oral Health Summit Framework. The $\mathrm{KOH}$ questionnaire was further reviewed and supported by the Director of the Masters Entry to Nursing Practice Program at DePaul University School of Nursing.

Internal consistency reliability of the $\mathrm{KOH}$ questionnaire was assessed using the Kuder-Richardson formula for Cronbach's alpha. All study participants answered questions one and ten correctly therefore the variance was zero. Cronbach's alpha for the remaining questions two through nine was 0.593 . Interestingly, removing question 4 resulted in an alpha of 0.703 , an acceptable internal consistency for a newly developed questionnaire.

\subsection{Data collection}

After receiving institutional review board approval for exempt review because no personal identifiers were used, the 
pretest, intervention, and posttest was conducted following the verbal recruitment statement as an in-service. An information sheet about participation in the study and the $\mathrm{KOH}$ pretest was distributed to interested participants with a notecard that had a random study code number between 000-999. All were informed that they were not required to participate in order to attend the in-service. They were asked to review the information sheet attached to the pretest about study participation and their rights as a research subject before they voluntarily completed the pretest. A folder was provided for consenting participants to return completed $\mathrm{KOH}$ pretests before the intervention and $\mathrm{KOH}$ posttests after the intervention identified by their provided study code. Participants were asked to discard their study code notecard outside of the library.

Data was collected at one in-service session to control the attrition rate. Also, to preserve consistency, the $\mathrm{KOH}$ pretest was conducted first, followed by the intervention, which was immediately followed by the $\mathrm{KOH}$ posttest during the one hour in-service period. Participants remained in the same room and seat and answered the questions individually. They were unable to take the test out of the room and unable to have conversation while participants were still taking either test. The researcher stepped out of the room for the duration of the tests and returned when the nurse educator indicated the tests were completed and turned into the closed folder.

\section{RESULTS}

\subsection{Data analysis}

Data coded by the random study code number was entered into SPSS Version 20 for analysis. Each test was linked to the respective nursing role (nurse assistant, licensed practical nurse, registered nurses and other: restorative nurse aide) as identified by the unscored item at the end of the $\mathrm{KOH}$ questionnaire. The test scores were determined by taking the number of questions answered correctly divided by the total number of questions (unanswered questions were scored as incorrect).

\subsubsection{Descriptive statistics}

Descriptive statistics including the mean and standard deviation were calculated for the total scores to determine the baseline knowledge of the nursing staff prior to the oral health education program intervention. $\mathrm{KOH}$ pretest scores formed a nearly symmetrical distribution where the mean = 5.40 , was close to and greater than the median and mode, both at 5.0. The range of correct answers was 3-8 of 10 questions. Kurtosis was -0.771 , indicating that the curve was platykurtic, but not significantly kurtosed as the standard deviation of \pm 1.569 was not exceeded. Skewness was 0.341 and between -1 to 1 , thus not significantly skewed.

Most participants improved their knowledge scores on the posttest except one nursing assistant received a lower score on the posttest and one licensed practical nurse earned the same score on the posttest. Descriptive statistics of the posttest scores were analyzed where the mean of 7.5 was slightly less than the median of 8. The range was 3-10 questions answered correctly. The negative skewness at -0.962 indicates a higher number of higher scores (mode $=9$ ) were achieved on the posttest.

\subsubsection{Comparing pretest and posttest scores}

The $\mathrm{KOH}$ posttest displayed overall higher scores $(\mathrm{M}=7.50$, $\mathrm{SD}=1.701)$ than the pretest scores $(\mathrm{M}=5.40, \mathrm{SD}=1.569)$. As shown in Table 2, the $\mathrm{KOH}$ score increased by an average of 2.10 points with $\mathrm{SD}=1.619$ and $95 \%$ confidence interval, 1.342, 2.858. The intervention effect was large and statistically significant, $t(19)=5.801, p<.001$.

Table 2. Analysis of pretest and posttest scores

\begin{tabular}{lllll}
\hline & Mean & N & Std. Deviation & Std. Error Mean \\
\hline KOH Pretest Questions Correct & 5.40 & 20 & 1.569 & .351 \\
KOH Posttest Questions Correct & 7.50 & 20 & 1.701 & .380 \\
\hline
\end{tabular}

\section{Discussion}

\subsection{Limitations and findings}

A limitation of the study was the small sample size as the sample was a function of those available to attend the oral health education program. Although a sample size less than $\mathrm{n}=30$ typically will not warrant parametric statistics, the objective was a preliminary analysis aimed at evaluating whether a difference exists in the oral health knowledge of nurses following participation in an oral health knowledge education program. The results indicated that study participants improved oral health knowledge and this improvement was statistically significant $(p<.05)$.

Additional attention should be given to increasing sample size and to increasing the diversity of participants as $75 \%$ of the participants studied were nursing assistants. The background oral health education and training of these participants was not known prior to the study. However, it is likely 
to be minimal, regardless of role certification, as reported by the USDHHS that little education of oral health and disease is taught in the curriculum of non-dental health professionals. $^{[10]}$

Knowledge change cannot be equated with behavior change. It remains unclear if the change in oral health knowledge demonstrated in this study can serve to improve the oral care of older people or promote oral self-care. Furthermore, the limiting factor of recent exposure to the knowledge presented in the program likely had a positive impact on the posttest scores. While it is recommended that there be longer intervals between a pretest and a posttest, this was not feasible as data collection was time limited.

\subsection{Implications for nursing practice}

Little time is devoted to oral health and disease topics in the education of non-dental health professionals ${ }^{[10]}$ such as the nursing staff population studied here. The nursing staff was capable of learning oral health knowledge necessary to provide care or promote oral self-care of their residents as evidenced by a statistically significant difference in $\mathrm{KOH}$ pretest and posttest scores. The findings of a $21 \%$ increase, from $54 \%$ to $75 \%$, are similar to findings by Mynors-Wallis and David ${ }^{[15]}$ that assessed oral health knowledge of nurses caring for older people using the Rak and Warren's ${ }^{[17]}$ questionnaire after an education intervention. By providing oral health education interventions, more non-dental health professionals, notably nursing staff, can improve the oral health of patients in their care.

Although not quantitatively measured, an improvement in the attitude about oral care was observed amongst the nursing staff that seemed to genuinely change their interest in oral health as some of the information provided during the oral health education program contradicted their previous learning. For example, the researcher fielded questions about the ADA recommendation to brush twice a day rather than after every meal. Staff asked questions about how oral health has systemic effects and subsequent cardiovascular diseases. Following the posttest, participants requested a review of the $\mathrm{KOH}$ questionnaire answers showing an interest in their understanding of the content. Some staff stayed after completion of the study to ask more questions about promoting oral care with disadvantaged older people that may appear resistant. Similarly to Frenkel, Harvey, and Needs, ${ }^{[20]}$ the researcher perceived improving positive attitudes towards oral health in addition to the difference in baseline postintervention oral health knowledge quantitatively analyzed.

Prior to conducting the study, the nurse educator informed the researcher that the staff at the nursing home for disadvan-

Published by Sciedu Press taged older people is working on putting oral care as a higher priority due to concerns about residents' teeth. These actions coincide with the nation's concern about the "epidemic" of poor oral health. Recent publications in popular media about poor oral health of Americans, notably older people, include New York Time's “In Nursing Homes, an Epidemic of Poor Dental Hygiene" [24] that highlights that oral health is not a priority in settings caring for older people. Support that oral health knowledge can be improved by educating nursing staff can help guide the definition of competency requirements for care of older people. The Code of Federal Regulations 483.25(a)(3) for nursing home regulation requires that oral health care be provided to older people if unable to care for themselves ${ }^{[15]}$ and policies and procedures must be improved to help nursing staff better care for the oral health of older people, whether or not they are economically-disadvantaged.

\section{Conclusion}

\section{Recommendations for further research}

Further refinement of the newly created instrument, the $\mathrm{KOH}$ questionnaire, and the oral health education program intervention should be priority. The internal consistency reliability of the $\mathrm{KOH}$ questionnaire was only considered acceptable after questions one and ten were removed because they accounted for zero variance. Additional questions should be added to improve variability and therefore improve reliability. Further, replication with a larger sample size can help validate the efficacy of the oral health education program and $\mathrm{KOH}$ questionnaire in improving oral health knowledge. There is also need to lengthen the time interval between the $\mathrm{KOH}$ pretest and posttest. Although results of the pilot study indicated that oral health knowledge improved after an oral health education program, data collection was time limited and the short one hour period for pretest, intervention, and posttest was too short to demonstrate longterm retention of knowledge. Further research is needed to evaluate the retention of oral health knowledge over a longer time span and to determine a relationship between improved knowledge and improved behavior of oral care or encouragement of oral self-care.

Although it is not appropriate to generalize adequate or improved oral health knowledge as equivalent to improved behaviors or oral care and promotion, the idea that knowledge as the basis for behavior is widely accepted and would apply in patient care areas internationally. Nursing has an integral role in health promotion ${ }^{[2]}$ therefore, it is imperative that nursing staff have sufficient oral health knowledge so that they understand the importance of providing oral care and promoting oral self-care. 


\section{ACKNOWLEDGEMENTS}

I wish to acknowledge my research advisor and co-author, Dr. Karyn Holm, and my course instructors, Dr. Elizabeth Florez and Dr. Matthew Sorenson for their valuable assistance and advice. Thank you to course instructor and friend, Ruth Tupper, MS, RN for her help with data analysis. Ad- ditionally, thank you to Anne Clancey for her review of the $\mathrm{KOH}$ questionnaire and to the Albert Schweitzer Fellowship for fueling my passion for oral health promotion and disease prevention of the underserved.

\section{CONFlicts OF InTEREST Disclosure}

The authors declare that there is no conflict of interest.

\section{REFERENCES}

[1] Petersen PE. The World Oral Health Report 2003: continuous improvement of oral health in the 21st century - the approach of the WHO Global Oral Health Programme. Community Dentistry \& Oral Epidemiology. 2003; 31(Suppl 1): 3-24. http://dx.doi.org/10. 1046/j . . 2003. com122.x

[2] National Academy of Sciences (US), Institute of Medicine and $\mathrm{Na}$ tional Research Council. Improving access to oral health care for vulnerable and underserved populations. Washington, D.C.: National Academies Press; 2011. Available from: http://www.nap.edu/ openbook.php?record_id $=13116$

[3] Petersen PE, Kandelman D, Arpin S, et al. Global oral health of older people-call for public health action. Community Dental Health. 2010; 27(Suppl 2): 257-68. Available from: http://www.ncbi.n lm.nih.gov/pubmed/?term=21313969 PMid:21313969

[4] Mariño R, Calache H, Morgan M. A community-based culturally competent oral health promotion for migrant older adults living in Melbourne, Australia. Journal of the American Geriatrics Society. 2013 Feb; 61(2): 270-5. PMid:23320643 http://dx . doi .org/10. $1111 /$ jgs. 12078

[5] Petersen PE, Yamamoto T. Improving the oral health of older people: the approach of the WHO Global Oral Health Programme. Community Dentistry \& Oral Epidemioly. 2005 Apr; 33(2): 81-92. http://dx.doi.org/10.1111/j.1600-0528.2004.00219.x

[6] Griffin SO, Jones JA, Brunson D, et al. Burden of oral disease among older adults and implications for public health priorities. American Journal of Public Health. 2012 Mar; 102(3): 411-8. http://dx.doi.org/10.2105/AJPH. 2011.300362

[7] Blinkhorn F, Weingarten L, Boivin L, et al. An intervention to improve the oral health of residents in an aged care facility led by nurses. Health Education Journal. 2011 Jun; 7(4): 9. http: //dx.doi.org/10.1177/0017896911412127

[8] Coleman P, Watson NM. Oral care provided by certified nursing assistants in nursing homes. Journal of American Geriatrics Society. 2006; 54(1): 138-43. PMid:16420211 http://dx.doi.org/10.1111/j $.1532-5415.2005 .00565 . x$

[9] Willis MS, Esqueda CW, Schacht RN. Social perceptions of individuals missing upper front teeth. Perceptual and Motor Skills. 2008 Apr; 106(2): 423-35. http://dx.doi.org/10.2466/pms.106.2 .423-435

[10] US Department of Health and Human Services. Oral health in America: A report of the surgeon general (executive summary) Rockville, MD: National Institute of Dental and Craniofacial Research of the National Institutes of Health. 2000. Available from: http://www.nidcr.nih.gov/DataStatistics/Surge onGeneral/Report/ExecutiveSummary.htm

[11] Wrightson AS, Stein PS. Smiles for life: an oral health education resource. Journal of the American Medical Directors Association. 2012 Oct; 13(8): 679-81. http://dx.doi.org/10.1016/j.jam da. 2012.07.012

[12] Locker D, Matear D, Stephens M, et al. Oral health-related quality 38 of life of a population of medically compromised elderly people.
Community Dental Health. 2002 Jun; 19(2): 90-7. Available from: http://www.ncbi.nlm.nih.gov/pubmed/12146588

[13] Macek MD, Manski MC, Schneiderman MT, et al. Knowledge of oral health issues among low-income Baltimore adults: a pilot study. Journal of Dental Hygiene. 2011 Winter; 85(1): 49-56. Available from: http://www.ncbi.nlm.nih.gov/pubmed/21396263

[14] Mynors-Wallis J, Davis DM. An assessment of the oral health knowledge and recall after a dental talk amongst nurses working with elderly patients: a pilot study. Gerodontology. 2004; 21(4): 201-4. http://dx.doi.org/10.1111/j.1741-2358.2004.00036.x

[15] Quality of Care, 42 C.F.R. Sect. 483.25. 2014. Available from: http://www.gpo.gov/fdsys/pkg/CFR-2011-title42 -vol5/pdf/CFR-2011-title42-vol5-sec483-25.pdf

[16] Gammack JK, Pulisetty S. Nursing education and improvement in oral care delivery in long-term care. Journal of the American Medical Directors Association. 2009 Nov; 10(9): 658-61. http: //dx.doi.org/10.1016/j.jamda.2009.09.001

[17] Rak OS, Warren K. An assessment of the level of dental and mouthcare knowledge amongst nurses working with elderly patients. Community Dental Health. 1990 Sep; 7(3): 295-301. PMid:2076506 Available from: http://www.ncbi.nlm.nih.gov/pubmed/2076 506

[18] Kullberg E, Forsell M, Wedel P, et al. Dental hygiene education for nursing staff. Geriatric Nursing. 2009 Sept-Oct; 30(5): 32933. PMid: 19818268 http://dx.doi.org/10.1016/j.gerinur se.2009.06.009

[19] Kullberg E, Sjögren P, Forsell M, et al. Dental hygiene education for nursing staff in a nursing home for older people. Journal of Advanced Nursing. 2010 Jun; 66(6): 1273-9. http://dx.doi.org/10.1111 /j.1365-2648.2010.05298.x

[20] Frenkel H, Harvey I, Needs K. Oral health care education and its effect on caregivers' knowledge and attitudes: a randomised controlled trial. Community Dentistry \& Oral Epidemiology. 2002 Apr; 30(2): 91-100. PMid:12000349 http://dx.doi.org/10.1034/j $.1600-0528.2002 .300202 . x$

[21] Parsons S. Collaborative oral health education for caregivers in an assisted-living facility. Journal of Nursing Education and Practice. 2013 Mar; 3(3): 44-51. http://dx.doi.org/10.5430/jnep.v3 n3p44

[22] Dolce MC. Nurse faculty enrichment and competency development in oral-systemic health. Nursing Research Practice [Internet]. 2012; 2012: 567058. http://dx.doi.org/10.1155/2012/567058

[23] Clark MB, Douglass AB, Maier R, et al. Smiles for Life: A National Oral Health Curriculum 3rd Edition [Internet]. Society of Teachers of Family Medicine; 2010. Available from: http://www.smiles forlifeoralhealth.com

[24] Saint Louis C. In Nursing Homes, an Epidemic of Poor Dental Hygiene. The New York Times. 2013. Available from: http://well.blogs.nytimes.com/2013/08/04/in-nursi ng-homes-an-epidemic-of-poor-dental-hygiene/?_r=0

ISSN 1925-4040 E-ISSN 1925-4059 